\title{
Idelalisib addition has neutral to beneficial effects on quality of life in bendamustine/ rituximab-treated patients: results of a phase 3 , randomized, controlled trial
}

Marco Montillo ${ }^{1 *}$, Árpád Illés ${ }^{2}$, Tadeusz Robak ${ }^{3}$, Alexander S. Pristupa ${ }^{4}$, Malgorzata Wach ${ }^{5}$, Miklós Egyed ${ }^{6}$, Julio Delgado ${ }^{7}$, Wojciech Jurczak ${ }^{8}$, Franck Morschhauser ${ }^{9}$, Anna Schuh $^{10}$, Herbert Eradat ${ }^{11}$, Sanatan Shreay ${ }^{12}$, Jacqueline C. Barrientos ${ }^{13}$ and Andrew D. Zelenetz ${ }^{14}$

\begin{abstract}
Background: In a phase 3 randomized, double-blind, placebo-controlled trial, treatment with idelalisib, a phosphoinositol-3 kinase $\delta$ inhibitor, + bendamustine/rituximab improved progression-free survival (PFS) and overall survival (OS) in adult patients with relapsed/refractory chronic lymphocytic leukemia (R/R CLL). Here we report the results of health-related quality of life (HRQL) analyses from this study.

Methods: From June 15, 2012 to August 21, 2014, 416 patients with R/R CLL were enrolled; 207 patients were randomized to the idelalisib arm and 209 to the placebo arm. In the 416 patients randomized to receive bendamustine/rituximab and either oral idelalisib $150 \mathrm{mg}$ twice-daily or placebo, HRQL was assessed at baseline and throughout the blinded part of the study using the Functional Assessment of Cancer Therapy-Leukemia (FACTLeu) and EuroQoL Five-Dimension (EQ-5D) visual analogue scale (VAS) questionnaires. The assessments were performed at scheduled patient visits; every 4 weeks for the first 6 months from the initiation of treatment, then every 8 weeks for the next 6 months, and every 12 weeks thereafter until end of study. Least-squares mean changes from baseline were estimated using a mixed-effects model by including treatment, time, and treatment-by-time interaction, and stratification factors as fixed effects. Time to first symptom improvement was assessed by KaplanMeier analysis.

Results: In mixed-effects model analysis, idelalisib + bendamustine/rituximab treatment led to clinically meaningful improvements from baseline in leukemia-associated symptoms. Moreover, per Kaplan-Meier analysis, the proportion of patients with symptom improvement was higher and time to improvement was shorter among patients in the idelalisib-containing arm compared with those who did not receive idelalisib. The physical and social/family FACTLeu subscale scores, along with the self-rated health assessed by EQ-VAS, showed improvement with idelalisib over placebo, but the difference did not reach statistical significance. The functional and emotional FACT-Leu subscale scores remained similar to placebo.
\end{abstract}

Conclusions: Addition of idelalisib to bendamustine/rituximab, apart from improving PFS and OS, had a neutral to beneficial impact on HRQL in patients with R/R CLL, particularly by reducing leukemia-specific disease symptoms.

Trial registration: Clinicaltrials.gov NCT01569295. Registered April 3, 2012.

Keywords: Idelalisib, Relapsed/refractory CLL, Patient-related outcomes, Health-related quality of life, Randomized phase 3 study

\footnotetext{
* Correspondence: marco.montillo@ospedaleniguarda.it

${ }^{1}$ Department of Hematology, Niguarda Cancer Center, Niguarda Hospital,

Piazza Ospedale Maggiore, 3, 20162 Milan, Italy

Full list of author information is available at the end of the article
}

(c) The Author(s). 2019 Open Access This article is distributed under the terms of the Creative Commons Attribution 4.0 International License (http://creativecommons.org/licenses/by/4.0/), which permits unrestricted use, distribution, and reproduction in any medium, provided you give appropriate credit to the original author(s) and the source, provide a link to the Creative Commons license, and indicate if changes were made. The Creative Commons Public Domain Dedication waiver (http://creativecommons.org/publicdomain/zero/1.0/) applies to the data made available in this article, unless otherwise stated. 


\section{Background}

In 2018, chronic lymphocytic leukemia (CLL) - the most common chronic leukemia-represented an estimated $1.2 \%$ of all newly diagnosed cancers in the US, with an estimated 20,940 new CLL cases and 4510 deaths [1]. The age-adjusted rate of CLL incidence is 4.7 per 100 , 000 persons per year. Owing to the introduction of new, improved treatment regimens, the 5-year relative survival rate increased from $65.5 \%$ in 1975 to $84.2 \%$ in 2014 [1]. Despite this improvement, CLL remains incurable, and the majority of patients experience disease relapse [2]. The risk of relapse is increased in patients with CLL-associated genomic aberrations [3-6], patients $\geq 65$ years with comorbid conditions [7-10], and refractory disease [11]. It is not uncommon for these patients to experience low treatment satisfaction and greatly reduced health-related quality of life (HRQL), mostly due to disease-related symptoms, toxicity of therapies, and anxiety associated with relapsing disease [12-14]. Despite recommendations to include prospective analyses of patient-reported outcomes (PROs) as additional endpoints in oncology clinical trials [15-17], PRO-based reports on HRQL in CLL are sparse. Patient-reported outcomes data can provide important information on the impact of new treatment regimens, including their efficacy and toxicity, as seen from the patient's perspective [16], and improvement in HRQL often reflects the efficacy of the new treatment under evaluation [18-21].

Idelalisib, in combination with rituximab, was approved for the treatment of patients with relapsed CLL for whom rituximab alone would be considered an appropriate therapy due to other comorbidities [22]. To examine the usefulness of idelalisib in additional clinical scenarios, we conducted a pivotal phase 3 , randomized, multicenter, double-blind, placebo-controlled trial of idelalisib, a phosphoinositol-3 kinase $\delta$ (PI3K $\delta$ ) inhibitor, in combination with bendamustine and rituximab, in patients with relapsed/refractory CLL (R/R CLL). The efficacy of the idelalisib/bendamustine/rituximab combination in this trial was superior to placebo/bendamustine/ rituximab, and substantially improved the primary endpoint of progression-free survival (PFS) as well as the key secondary endpoint of overall survival (OS) [23].

Prespecified exploratory endpoints in this study included assessment of HRQL using the Functional Assessment of Cancer Therapy - Leukemia (FACT-Leu) validated questionnaire [24-28]. The FACT-Leu was developed as a disease-specific HRQL questionnaire for patients with leukemia $[27,28]$, and is composed of subscales scoring the patient's physical, functional, social/ family, and emotional well-being, as well as leukemiaspecific disease symptoms [27, 28]. Another prespecified exploratory endpoint was global health status and selfrated health assessed using the EuroQoL Five-Dimension
(EQ-5D) visual analogue scale (VAS) questionnaires $[29,30]$. We present the results of these prospectively defined analyses comparing the impact of treatment with idelalisib combined with bendamustine/rituximab with that of bendamustine/rituximab/placebo, on patients' HRQL.

\section{Methods \\ Study design and participants}

This was a phase 3, randomized, multicenter, doubleblind, placebo-controlled study (NCT01569295) in adult patients who were diagnosed with CLL requiring treatment according to International Workshop on Chronic Lymphocytic Leukemia criteria [17]. Eligible patients had measurable lymphadenopathy by computed tomography or magnetic resonance imaging, received prior therapy containing a purine analog or bendamustine and an anti-CD20 monoclonal antibody, experienced CLL progression within $<36$ months since completion of the last prior therapy, were eligible to receive cytotoxic therapy, and had a Karnofsky Performance Status score of $\geq 60$. Study design and detailed eligibility criteria were published previously [23].

\section{Ethics, consent, and permissions}

The study protocols were approved by the Institutional Review Boards at each study site. The trial was conducted according to the principles of Good Clinical Practice and the Declaration of Helsinki. All patients provided written informed consent.

\section{Treatments and endpoints}

Patients received oral idelalisib $150 \mathrm{mg}$ or matching placebo twice daily. Bendamustine $70 \mathrm{mg} / \mathrm{m}^{2}$ was administered intravenously on days 1 and 2 for six 28-day cycles in both arms. Rituximab was administered intravenously with each cycle of bendamustine at $375 \mathrm{mg} / \mathrm{m}^{2}$ on day 1 of cycle 1 , and $500 \mathrm{mg} / \mathrm{m}^{2}$ on day 1 of cycles 2 to 6 . Idelalisib/placebo was given continuously, until disease progression, death, or intolerable toxicity. Bendamustine and rituximab were administered for up to a maximum of 12 and 6 infusions, respectively [23].

The details on primary and secondary endpoints have been reported [23]. Briefly, the primary endpoint was PFS; OS, overall response rate, and safety were among the key secondary endpoints. The prespecified HRQLrelated exploratory endpoints were change from baseline in HRQL domain and symptom scores based on the FACT-Leu, and change from baseline in overall health and single-item dimension scores from the EQ-5D and EQ-VAS questionnaires. Key time points were the end of the randomized, double-blind initial period of combination therapy with bendamustine/rituximab at week 24 and the end of the preplanned continuing therapy period with idelalisib or placebo alone at week 48 . 


\section{HRQL assessments}

Patient well-being was assessed using the FACT-Leu questionnaire composed of 44 items measuring physical well-being (PWB, 7 items), functional well-being (FWB, 7 items), social/family well-being (S/FWB, 7 items), emotional well-being (EWB, 6 items), and leukemiaspecific concerns (LeuS, 17 items) [28], scored based on the Functional Assessment of Chronic Illness Therapy-3 scoring guideline and user manual [31]. The subscale scores represent the sums of each individual item score. The composite scores include FACT-Leu total score (range 0-176), which is the sum of all subscales, and the Trial Outcome Index (TOI, range 0-124), which is the sum of the PWB, FWB, and LeuS subscales. Higher scores are associated with better self-reported HRQL.

The EQ-5D questionnaire contains 5 dimensions: mobility, self-care, usual activities, pain/discomfort, and anxiety/depression. Each dimension has 3 levels: no problems, some problems, and extreme problems [32]. The EQ-5D is converted into a single utility index, designed as an international, standardized questionnaire for evaluation of HRQL [29, 30], by applying the US preference-weighted index [33]. The EQ-VAS is used to assess patient's self-rated health on a $100-\mathrm{mm}$ scale ranging from "Worst imaginable health state" to "Best imaginable health state." The EQ-5D utility and EQ-VAS scores are considered reliable and valid for assessing HRQL in cancer patients [34]. Positive changes from baseline indicate improvement in HRQL.

The surveys were administered every 4 weeks for the first 6 months from the initiation of treatment, then every 8 weeks for the next 6 months, and every 12 weeks thereafter until the end of study or until the patient was no longer receiving blinded study drug for any reason.

\section{HRQL statistical analyses}

The FACT Leu and EQ-5D questionnaire was scored and processed according to the user manual [32]. The HRQL questionnaire compliance was defined as a patient having answered at least 1 question at an assessment time point. The compliance rates for each study arm and at each time point were calculated as the number of patients who completed at least 1 question divided by the total number of patients available at that assessment time point. The frequency and proportion of reported problems for each of the five EQ-5D dimensions were summarized at each scheduled assessment. For the FACT-Leu, EQ-VAS questionnaires, and EQ-5D utility index, least-squares mean changes from baseline were estimated using a mixed-effects model, by including treatment, time, and treatment-by-time interaction, and stratification factors as fixed effects. Mixed-effects model used all available data up to week 84 , as $<10 \%$ of placebo patients have data available beyond week 84 . The least-squares means of change from baseline over time were plotted. The minimally important difference (MID) ranges were defined for the different subscales and are summarized in Additional file 1: Table S1. The lower bound of the MID range was utilized when defining symptom improvement; an increase of at least 3 points from baseline for PWB, S/FWB, FWB, and EWB, and 5 points for LeuS (reaching MID). Time to first symptom improvement was analyzed by the KaplanMeier (KM) method. The hazard ratios with 95\% confidence intervals (CIs) were estimated from a Cox proportional hazards model without any adjustment and $P$-value from log-rank test was reported to examine the difference between the 2 treatment arms.

The HRQL analyses were based on the intent-to-treat analysis set, which included all patients randomized

Table 1 Scores for the HRQL questionnaires at baseline

\begin{tabular}{|c|c|c|c|c|}
\hline & Idelalisib/rituximab/bendamustine, $N=207$ & $n$ & Placebo/rituximab/bendamustine, $N=209$ & $\mathrm{n}$ \\
\hline$\overline{\text { FACT-Leu total score }}{ }^{\mathrm{a}}$ & $125.27(24.103)$ & 196 & $123.17(27.540)$ & 202 \\
\hline Trial outcome index score ${ }^{b}$ & $86.12(18.662)$ & 196 & $84.71(21.337)$ & 203 \\
\hline Physical well-being & $21.77(5.012)$ & 197 & $21.39(5.418)$ & 203 \\
\hline Social/family well-being & $21.51(5.502)$ & 198 & $21.40(5.392)$ & 203 \\
\hline Emotional well-being & $17.68(4.215)$ & 198 & $16.93(4.902)$ & 204 \\
\hline Functional well-being & $17.99(6.068)$ & 199 & $17.32(6.135)$ & 204 \\
\hline Leukemia-specific symptoms & $46.31(10.312)$ & 199 & $45.95(12.206)$ & 204 \\
\hline EQ-5D utility index & $0.78(0.217)$ & 197 & $0.78(0.228)$ & 195 \\
\hline EQ-VAS & $68.8(17.81)$ & 190 & $67.4(19.28)$ & 194 \\
\hline
\end{tabular}

Analyzed in the ITT population. All data presented as mean (SD); $\mathrm{n}$ indicates number of patients available for $\mathrm{HRQL}$ assessment at baseline

EQ-5D EuroQoL Five-Dimension, FACT-Leu functional assessment of cancer therapy-leukemia, EWB emotional well-being, FWB functional well-being, HRQL healthrelated quality of life, ITT intent-to-treat, LeuS leukemia-specific concerns, PWB physical well-being, S/FWB social/family well-being, SD standard deviation, TOI trial outcome index, VAS visual analog scale

${ }^{\mathrm{a}} \mathrm{FACT}$-Leu Total $=$ LeuS $+\mathrm{PWB}+\mathrm{S} / \mathrm{FWB}+\mathrm{EWB}+\mathrm{FWB}$

${ }^{\mathrm{b}} \mathrm{TOI}=\mathrm{LeuS}+\mathrm{PWB}+\mathrm{FWB}$ 
a

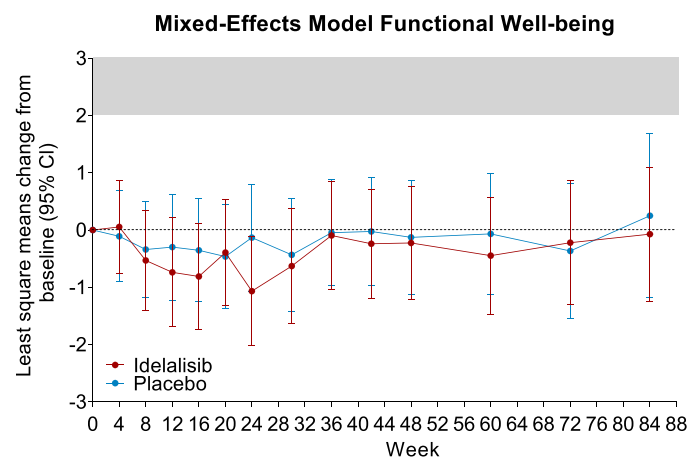

C

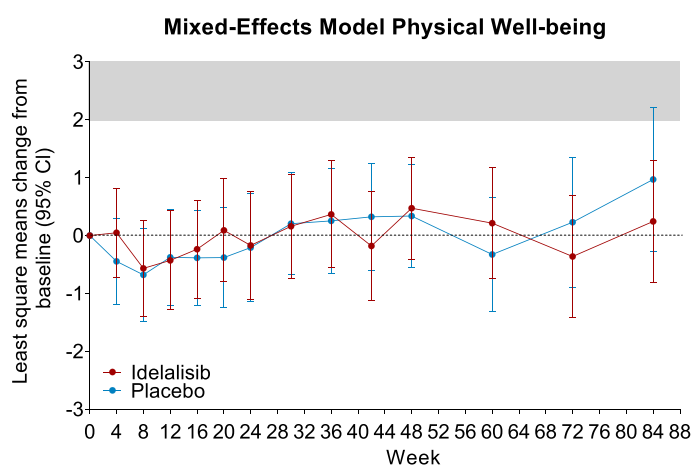

e

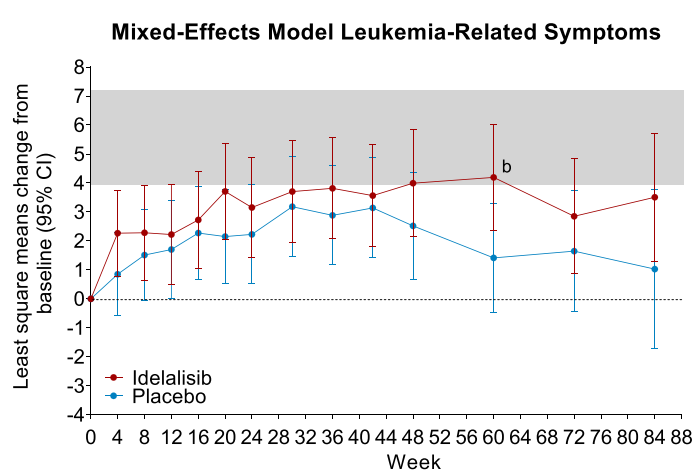

g

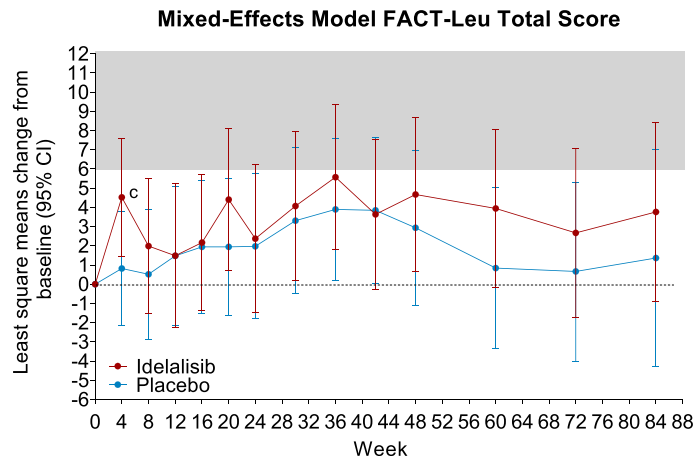

Fig. 1 (See legend on next page.) b

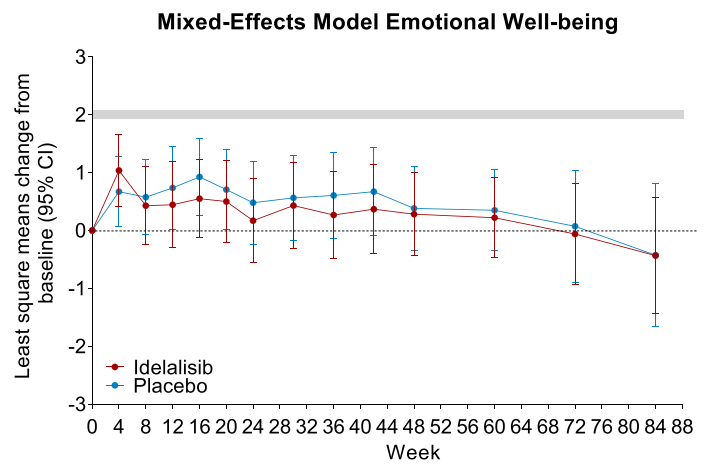

d Mixed-Effects Model Social/Family Well-being

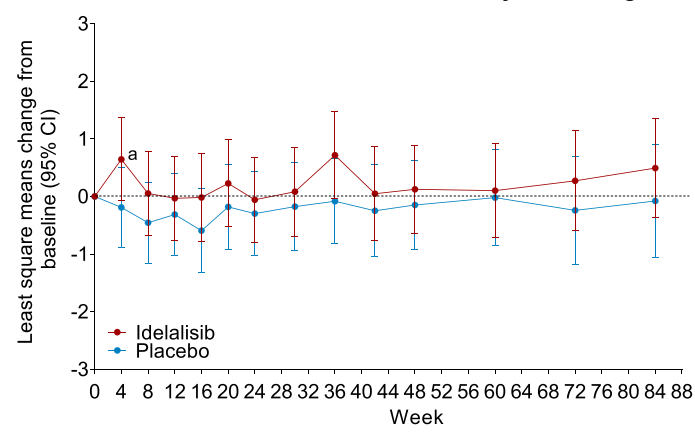

f

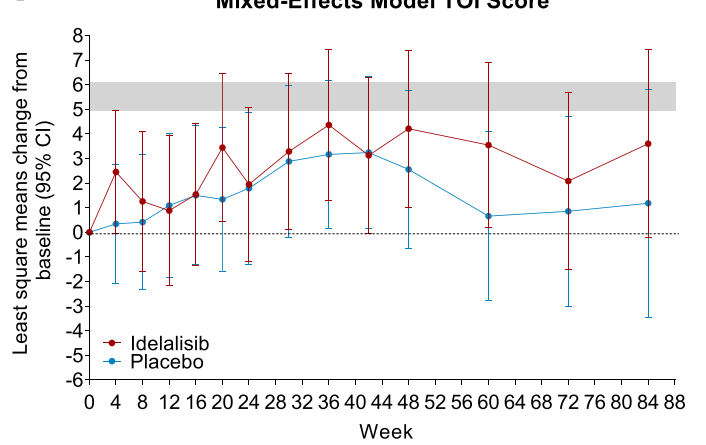


(See figure on previous page.)

Fig. 1 Mixed-effects model analysis of FACT-Leu. a, FWB; $\mathbf{b}$, EWB; $\mathbf{c}$, PWB; $\mathbf{d}$, S/FWB; $\mathbf{e}$, LeuS; $\mathbf{f}$, TOI score; $\mathbf{g}$, FACT-Leu total score. Curves above the $x$-axis indicate positive effects, and curves below the axis show negative effects. Gray area denotes MID range. ${ }^{a} P=0.0525$ for treatment difference. ${ }^{b} P=0.0192$ for treatment difference. ${ }^{C} P=0.0343$ for treatment difference. $C l$ confidence interval, EWB emotional well-being, $F A C T$-Leu Functional Assessment of Cancer Therapy-Leukemia, FWB functional well-being, LeuS leukemia-specific symptoms, MID minimally important difference, PWB physical well-being, S/FWB social/family well-being, TO/ trial outcome index

in the study regardless of whether study drug was administered, and with treatment groups designated according to initial randomization. Nominal $P$-value threshold of 0.05 was used for significance testing without multiplicity adjustment. All the analyses were performed using SAS version 9.2 (Cary, North Carolina).

\section{Results}

\section{Key patient characteristics}

From June 15, 2012, to August 21, 2014, 416 patients with R/R CLL were enrolled; 207 patients were randomized to the idelalisib arm and 209 to the placebo arm. Based on the results of the formal interim analysis performed in June 2015, the Independent Data Monitoring Committee recommended study unblinding. Although the study is still ongoing, the HRQL data presented herein reflect a data cutoff date of October 7, 2015, at the time of study unblinding. At the time of this analysis, 141 (34\%) of 416 patients were continuing in the study; 95 (46\%) of 207 in the idelalisib group and 46 (22\%) of 209 in the placebo group.

Patient disposition and demographic and baseline characteristics were published previously [23]. The patients received a median (quartile [Q1, Q3]) of $6(4$, 6) cycles of treatment with bendamustine and $6(5,6)$ with rituximab; the median $(\mathrm{Q} 1, \mathrm{Q} 3)$ exposure to idelalisib and placebo was $14.8(5.9,18.9)$ months and $11.1(5.8,15.3)$ months, respectively. Generally, demographic and baseline disease characteristics were balanced across the treatment groups [23].

\section{Summary of the efficacy and safety results}

The detailed efficacy and safety results were previously reported at the data cutoff of October 7, 2015 [23], the same as the present analysis. The addition of idelalisib to bendamustine/rituximab led to a substantial improvement in the efficacy of the treatment, compared with bendamustine/rituximab alone [23]. The most common all-grade adverse events (AEs) were neutropenia and pyrexia in the idelalisib arm and neutropenia and nausea in the placebo arm [23].

\section{Patient-reported FACT-Leu and EQ-5D outcomes}

Among patients randomized to idelalisib and placebo arms, similar numbers of patients were available for HRQL analyses at baseline (Table 1). Mean baseline scores for the FACT-Leu and EQ-5D questionnaires were also comparable between the treatment arms (Table 1).

Assessments of compliance for the FACT-Leu and EQ-5D questionnaires were conducted over the initial

Table 2 Summary of symptom improvement

\begin{tabular}{|c|c|c|c|c|c|c|c|c|}
\hline & \multicolumn{3}{|c|}{ Idelalisib/bendamustine/rituximab, N = 207} & \multicolumn{5}{|c|}{ Placebo/bendamustine/rituximab, N = 209} \\
\hline & $\begin{array}{l}\text { Patients with } \\
\text { MID } \\
\text { improvement }^{\mathrm{a}}\end{array}$ & $\begin{array}{l}\text { Time to } \\
\text { symptom } \\
\text { improvement }^{b}\end{array}$ & $\begin{array}{l}\text { Proportion of patients } \\
\text { with any symptom } \\
\text { improvement }^{c}\end{array}$ & $\begin{array}{l}\text { Patients with } \\
\text { MID } \\
\text { improvement }^{\mathrm{a}}\end{array}$ & $\begin{array}{l}\text { Time to } \\
\text { symptom } \\
\text { improvement }^{\mathrm{b}}\end{array}$ & $\begin{array}{l}\text { Proportion of patients } \\
\text { with any symptom } \\
\text { improvement }^{c}\end{array}$ & $\begin{array}{l}\text { HR } \\
(95 \% \mathrm{Cl})\end{array}$ & $P$-value \\
\hline PWB & $97(69.3)$ & $\begin{array}{l}12.3(9.1,16.1) \\
N=140\end{array}$ & $139(67.1)$ & $89(61.8)$ & $\begin{array}{l}20.9(12.9,30.1) \\
N=144\end{array}$ & $141(67.5)$ & $1.28(0.96,1.70)$ & 0.1026 \\
\hline S/FWB & $82(59.0)$ & $\begin{array}{l}20.4(12.1,39.9) \\
N=139\end{array}$ & 130 (62.8) & $79(52.7)$ & $\begin{array}{l}32.4(16.3,72.7) \\
N=150\end{array}$ & 139 (66.5) & $1.20(0.88,1.63)$ & 0.2663 \\
\hline EWB & $99(62.7)$ & $\begin{array}{l}16.1(8.9,23.9) \\
N=158\end{array}$ & $159(76.8)$ & $103(61.7)$ & $\begin{array}{l}16.9(12.4,24.4) \\
N=167\end{array}$ & 147 (70.3) & $1.04(0.79,1.37)$ & 0.8357 \\
\hline FWB & $102(60.0)$ & $\begin{array}{l}20.9(12.1,39.9) \\
N=170\end{array}$ & 142 (68.6) & $100(55.2)$ & $\begin{array}{l}24.7(16.1,44.3) \\
N=181\end{array}$ & $145(69.4)$ & $1.07(0.81,1.42)$ & 0.6321 \\
\hline LeuS & $142(74.7)$ & $\begin{array}{l}8.4(6.3,12.7) \\
N=190\end{array}$ & $168(81.2)$ & $133(68.6)$ & $\begin{array}{l}12.3(11,16.3) \\
N=194\end{array}$ & $168(80.4)$ & $1.22(0.96,1.55)$ & 0.1134 \\
\hline
\end{tabular}

Analyzed in the ITT population. Patients with baseline PWB/S/FWB/FWB $>25$, EWB $>21$, and LeuS $>63$ are not included in the respective analysis of improvement $C I$ confidence interval, EWB emotional well-being, FWB functional well-being, HRQL health-related quality of life, $H R$ hazard ratio, ITT intent-to-treat, LeuS leukemiaspecific concerns, MID minimally important difference, PWB physical well-being, S/FWB social/family well-being

${ }^{a}$ Data presented as $n(\%)$. MID symptom improvement was defined as an increase of $\geq 3$ points from baseline for PWB/S/FWB/FWB/EWB and 5 points for LeuS

${ }^{b}$ Data presented as median $(95 \% \mathrm{Cl})$, weeks. Patients who did not experience a symptom improvement compared to baseline were censored at their last available $\mathrm{HRQL}$ assessment time. Time to symptom improvement (weeks) $=($ date of first symptom improvement - date of randomization +1$) / 7$

${ }^{\mathrm{c}}$ Data presented as $\mathrm{n}(\%)$. Patients with any increase from baseline 


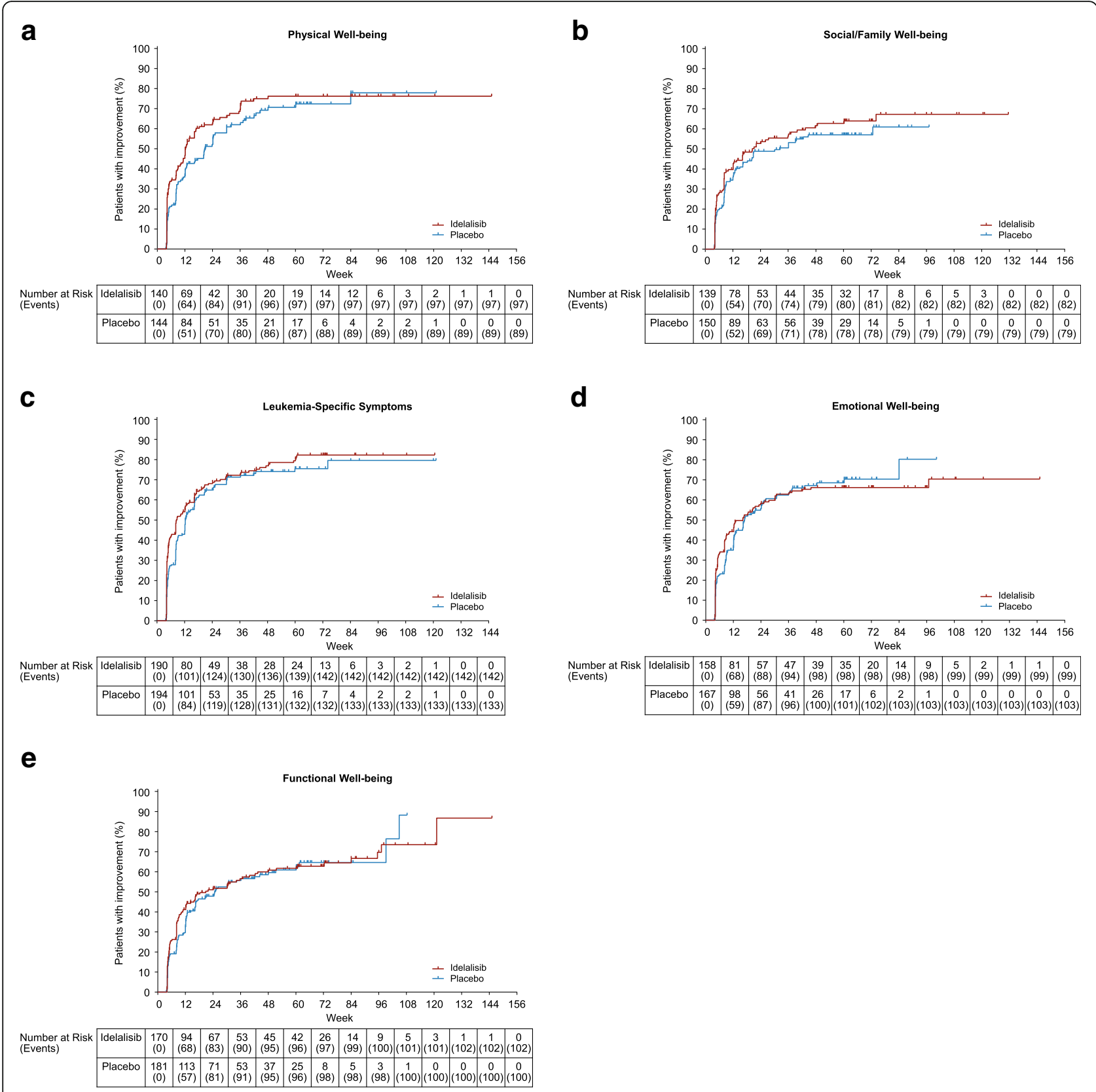

Fig. 2 Percent of patients with improvements in FACT-Leu subscales (Kaplan-Meier analysis). a, PWB; b, S/FWB; c, LeuS; d, EWB; e, FWB. EWB emotional well-being, FACT-Leu Functional Assessment of Cancer Therapy-Leukemia, FWB functional well-being, LeuS leukemia-specific symptoms, PWB physical well-being, S/FWB social/family well-being

144 weeks of study. Compliance rates for both FACTLeu and EQ-5D were high and exceeded $80 \%$ during the first 120 weeks of study in both treatment arms (Additional file 1: Tables S2 and S3).

\section{FACT-Leu}

The addition of idelalisib to bendamustine/rituximab had no significant impact on FWB, EWB, and PWB compared with placebo/bendamustine/rituximab (Fig. 1a, b, c). In contrast, idelalisib increased the S/FWB and
LeuS subscale scores, as well as composites TOI and FACT Leu total score, compared with the placebo arm, indicating an improvement in HRQL in idelalisib-treated patients (Fig. 1d, e, f, g). In a mixed-effects model including treatment arm (idelalisib vs placebo) and duration, treatment arm had no significant fixed effect on any FACT-Leu score. The effect of treatment duration was significant for FACT-Leu total score $(P=0.0076)$, TOI score $(P=0.0103)$, PWB $(P=0.0017)$, EWB $(P=$ $0.0142)$, and LeuS $(P=0.0023)$; there was no significant 
Table 3 Summary of EQ-5D questionnaire by dimension

\begin{tabular}{|c|c|c|c|c|c|c|}
\hline \multirow[b]{2}{*}{ Dimensions } & \multicolumn{3}{|c|}{ Idelalisib/rituximab/bendamustine, $N=207$} & \multicolumn{3}{|c|}{ Placebo/rituximab/bendamustine, $N=209$} \\
\hline & Baseline & Week 24 & Week 48 & Baseline & Week 24 & Week 48 \\
\hline \multicolumn{7}{|c|}{ Anxiety/Depression } \\
\hline Level 1 & $113(57.1)$ & $102(65.8)$ & $85(66.4)$ & $117(59.1)$ & $91(60.3)$ & $70(66.7)$ \\
\hline Level 2 & $84(42.4)$ & $50(32.3)$ & $41(32.0)$ & $75(37.9)$ & $55(36.4)$ & $32(30.5)$ \\
\hline Level 3 & $1(0.5)$ & $3(1.9)$ & $2(1.6)$ & $6(3.0)$ & $5(3.3)$ & $3(2.9)$ \\
\hline \multicolumn{7}{|l|}{ Mobility } \\
\hline Level 1 & $145(73.6)$ & $119(76.8)$ & $94(73.4)$ & $142(71.4)$ & $112(74.7)$ & $84(80.0)$ \\
\hline Level 2 & $52(26.4)$ & $36(23.2)$ & $34(26.6)$ & $55(27.6)$ & $38(25.3)$ & $21(20.0)$ \\
\hline Level 3 & 0 & 0 & 0 & $2(1.0)$ & 0 & 0 \\
\hline \multicolumn{7}{|c|}{ Pain/Discomfort } \\
\hline Level 1 & $105(53.3)$ & 96 (61.9) & $77(60.2)$ & $114(57.0)$ & 79 (52.3) & $63(60.6)$ \\
\hline Level 2 & $85(43.1)$ & $54(34.8)$ & $51(39.8)$ & $81(40.5)$ & $69(45.7)$ & $37(35.6)$ \\
\hline Level 3 & $7(3.6)$ & $5(3.2)$ & 0 & $5(2.5)$ & $3(2.0)$ & $4(3.8)$ \\
\hline \multicolumn{7}{|l|}{ Self-Care } \\
\hline Level 1 & $184(92.9)$ & $136(87.7)$ & 115 (89.8) & $184(92.5)$ & 131 (86.8) & $92(87.6)$ \\
\hline Level 2 & $14(7.1)$ & $19(12.3)$ & $13(10.2)$ & $13(6.5)$ & $20(13.2)$ & $12(11.4)$ \\
\hline Level 3 & 0 & 0 & 0 & $2(1.0)$ & 0 & $1(1.0)$ \\
\hline \multicolumn{7}{|c|}{ Usual Activities } \\
\hline Level 1 & $126(63.6)$ & $93(60.4)$ & $83(64.8)$ & $122(61.3)$ & 87 (57.6) & $71(68.3)$ \\
\hline Level 2 & 65 (32.8) & $52(33.8)$ & $45(35.2)$ & $70(35.2)$ & $61(40.4)$ & $31(29.8)$ \\
\hline Level 3 & $7(3.5)$ & $9(5.8)$ & 0 & $7(3.5)$ & $3(2.0)$ & $2(1.9)$ \\
\hline
\end{tabular}

Analyzed in the ITT population. All data represented as $\mathrm{n}(\%)$

Level 1: no problems; Level 2: some problems; Level 3: extreme problems

EQ-5D EuroQoL Five-Dimension, ITT intent-to-treat

interaction between treatment arm and duration for any FACT-Leu score. The treatment difference for idelalisib vs placebo was statistically significant for the LeuS subscale at week $60(P=0.0192)$, and for S/FWB and FACT-Leu total score at week $4(P=0.0525$ and $P=$ 0.0343 , respectively) (Fig. 1, Additional file 1: Table S4). The least squares mean changes from baseline in the LeuS subscale scores were within the MID range of 4 to 7 during the period between week 48 and week 60 in the idelalisib arm, while the values in the placebo arm did not reach the MID at any time points (Fig. 1e).

The KM analysis of symptom improvement suggests that an increased number of patients with the MID improvement, as well as shorter time to symptom improvement, were reported for the 5 FACT-Leu subscales in the idelalisib-containing arm compared with the placebo arm, but none of the differences reached statistical significance (Table 2). A higher proportion of patients treated with idelalisib achieved improvement in PWB, S/ FWB and LeuS subscale scores, but not EWB and FWB scores, compared with patients treated with placebo (Fig. 2a-e). Overall, addition of idelalisib to bendamustine/rituximab had a neutral to numerically favorable effect on change from baseline in FACT-Leu scores of patients with R/R CLL.

\section{EQ-5D}

Proportions of patients reporting level 1 or 2 EQ-5D health scores at weeks 24 and 48 were generally similar between the idelalisib and placebo treatment arms. Slightly fewer idelalisib-treated patients reported extreme problems (level 3) with anxiety/depression and pain/discomfort at baseline and after 24 and 48 weeks of treatment compared with patients who received placebo (Table 3). The EQ-5D utility index and EQ-VAS showed trends toward improvement with idelalisib relative to placebo treatment, but these did not reach significance (Table 4, Fig. 3). In a mixed-effects model including treatment (idelalisib vs placebo) and treatment duration, treatment duration had a significant fixed effect for EQ$5 \mathrm{D}$ utility index $(P=0.0169)$ and EQ-VAS $(P=0.0061)$; treatment had no significant fixed effect on either score, but there was a significant fixed effect of the interaction between treatment and treatment duration on EQ-5D utility index $(P=0.0395)$. Combination therapy with idelalisib vs placebo did not worsen patient-reported 
Table 4 Mixed-effects model analysis for functional assessment of cancer therapy using EQ-5D in the ITT population

\begin{tabular}{|c|c|c|}
\hline \multicolumn{3}{|c|}{ Treatment difference ${ }^{a}$ LSM (95\% CI) } \\
\hline & EQ-5D UI & EQ-VAS \\
\hline Week 4 & $0.03(-0.01,0.07)$ & $0.18(-3.24,3.61)$ \\
\hline$P$-value & 0.1302 & 0.9167 \\
\hline Week 8 & $-0.01(-0.05,0.04)$ & $-1.54(-5.06,1.98)$ \\
\hline$P$-value & 0.7570 & 0.3895 \\
\hline Week 12 & $0.00(-0.04,0.05)$ & $-1.00(-4.96,2.97)$ \\
\hline$P$-value & 0.8730 & 0.6216 \\
\hline Week 16 & $0.02(-0.03,0.07)$ & $-0.09(-3.82,3.65)$ \\
\hline$P$-value & 0.4325 & 0.9631 \\
\hline Week 20 & $0.02(-0.03,0.06)$ & $0.17(-3.74,4.09)$ \\
\hline$P$-value & 0.4514 & 0.9301 \\
\hline Week 24 & $0.04(-0.01,0.08)$ & $-0.04(-3.92,3.85)$ \\
\hline$P$-value & 0.1433 & 0.9851 \\
\hline Week 30 & $0.03(-0.02,0.08)$ & $0.69(-3.36,4.73)$ \\
\hline$P$-value & 0.2915 & 0.7389 \\
\hline Week 36 & $0.02(-0.03,0.07)$ & $1.94(-2.24,6.13)$ \\
\hline$P$-value & 0.4630 & 0.3623 \\
\hline Week 42 & $0.00(-0.06,0.06)$ & $-1.56(-5.92,2.80)$ \\
\hline$P$-value & 0.9995 & 0.4823 \\
\hline Week 48 & $0.02(-0.04,0.07)$ & $1.99(-2.06,6.03)$ \\
\hline$P$-value & 0.5601 & 0.3348 \\
\hline Week 60 & $0.03(-0.03,0.09)$ & $1.53(-3.05,6.11)$ \\
\hline$P$-value & 0.2981 & 0.5128 \\
\hline Week 72 & $0.04(-0.04,0.11)$ & $-0.97(-6.41,4.48)$ \\
\hline$P$-value & 0.3131 & 0.7275 \\
\hline Week 84 & $-0.04(-0.11,0.02)$ & $0.03(-6.14,6.20)$ \\
\hline$P$-value & 0.2039 & 0.9921 \\
\hline
\end{tabular}

Cl confidence interval, EQ-5D EuroQoL Five-Dimension, Ul utility index, ITT intent-to-treat, $L S M$ least squares means, VAS visual analog scale

health-and resulted in favorable trends in some measures-in patients with R/R CLL treated with bendamustine/rituximab.

\section{Discussion}

In the primary analysis, the addition of idelalisib to bendamustine/rituximab resulted in superior PFS and OS compared with bendamustine and rituximab alone, thus providing a substantial clinical benefit to R/R CLL patients - a patient population that is difficult to treat [23]. Apart from treatment goals, a more holistic understanding of the impact of treatment including patients' quality of life is also important, and HRQL data constitutes an important part of treatment evaluation. Because patients in both the idelalisib and placebo arms received concurrent chemo-immunotherapy with associated side effects, idelalisib could not be expected to substantially improve quality of life (QoL) compared with placebo. However, there was potential concern that addition of idelalisib to bendamustine/rituximab would adversely affect change in QoL relative to treatment with bendamustine/rituximab alone.

These prospectively defined HRQL analyses indicate that idelalisib in combination with bendamustine/rituximab treatment had a neutral impact on several HRQL domains in patients with R/R CLL compared with bendamustine/rituximab alone. Per mixed-effects model analyses, the addition of idelalisib to bendamustine/rituximab did not affect the FWB and EWB subscale scores, but provided minor improvements in the PWB, S/FWB, and LeuS subscale scores as well as the FACTLeu composite measures TOI and FACT-Leu total score. Increased LeuS subscale scores in the idelalisib arm were within the MID range at weeks 48 and 60 and reached statistical significance at week 60 . The KM analysis of the symptom improvement showed that among idelalisibtreated patients, time to symptom improvement was shortened by approximately 4 to 12 weeks for the LeuS, PWB, and S/FWB subscales, compared with the placebo arm, and a higher proportion of patients had clinically meaningful improvements in these HRQL domains.

Idelalisib treatment led to small numerical improvements in HRQL in the global health status, as evidenced by the EQ-5D results, particularly reduction of the number of patients who perceived themselves to experience "extreme problems" within the anxiety/depression and pain/discomfort dimensions. The mean changes from baseline in self-rated health, as assessed by EQ-VAS, was also improved, and reached statistical significance vs placebo at week 36 when patients had stopped concurrent chemo-immunotherapy. The rather small differences in overall quality of life observed between the 2 treatment arms may be due to side effects from the concurrent chemo-immunotherapy exerting a dominant effect on quality of life measurements. However, even the slight QoL improvement in patients treated with idelalisib vs placebo in addition to bendamustine/rituximab is valuable information considering the AEs recorded in previous clinical trials of idelalisib [23, 35, 36].

Recently published analyses of PROs assessed in phase 3 randomized trials in patients with relapsed CLL reported that ibrutinib in combination with bendamustine/rituximab vs bendamustine/rituximab alone neither improved nor adversely impacted HRQL [37], and small positive improvements were noted with the addition of ofatumumab to chlorambucil [38] or to fludarabine and cyclophosphamide [21]. However, apart from the fact that comparisons of the results across different studies have several limitations, even indirect comparison of these findings with the results from our study is difficult because of different tools used for PRO evaluations (The Functional 


\section{Mixed-Effects Model VAS}

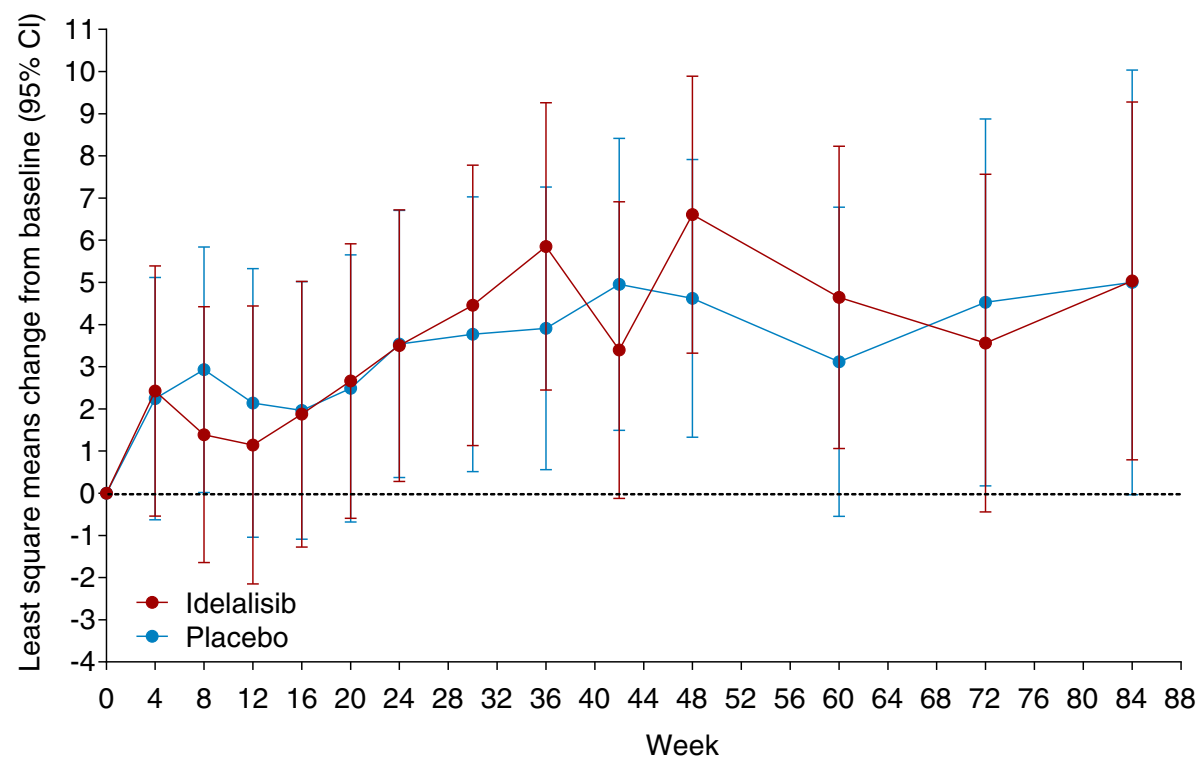

Fig. 3 Mixed-effects model analysis of EuroQoL Five-Dimension VAS. CI confidence interval, VAS visual analogue scale

Assessment of Chronic Illness Therapy - Fatigue scale and European Organisation for Research and Treatment of Cancer Quality of Life Questionnaire, in the ibrutinib and ofatumumab studies, and FACT-Leu in this study).

\section{Conclusion}

In summary, in patients with incurable diseases such as CLL, treatment efficacy needs to be balanced with safety and HRQL. In this study, we found that adding idelalisib to bendamustine/rituximab-even for those patients already treated with a prior bendamustine-containing regimen-improved treatment efficacy with, importantly, a neutral or beneficial impact on patients' HRQL. These HRQL findings provide additional information to balance the improved efficacy of the combination regimen with possible safety concerns reported with idelalisib use [23]. Overall, these results further support the existing body of evidence indicating that idelalisib treatment benefits patients with R/R CLL.

\section{Supplementary information}

Supplementary information accompanies this paper at https://doi.org/10. 1186/s12955-019-1232-8.

Additional file 1: Table S1. Questionnaires used to assess healthrelated quality of life. Table S2. Compliance rates: FACT-Leu questionnaire. Table S3. Compliance rates: EQ-5D questionnaire. Table S4. Mixed-effects model analysis estimates (idelalisib/placebo) for functional assessment of cancer therapy using FACT-Leu.

\section{Abbreviations}

AE: Adverse event; Cl: Confidence interval; CLL: Chronic lymphocytic leukemia; EQ-5D: EuroQoL Five-Dimension; EWB: Emotional well-being; FACT-
Leu: Functional assessment of cancer therapy - leukemia; FWB: Functional well-being; HR: Hazard ratio; HRQL: Health-related quality of life; KM: KaplanMeier; LeuS: Leukemia-specific concerns; MID: Minimally important difference; OS: Overall survival; PFS: Progression-free survival; PI3KS: Phosphoinositol-3 kinase $\delta$; PRO: Patient-reported outcomes; PWB: Physical well-being; Q: Quartile; QoL: Quality of life; R/R: Relapsed/refractory; S/FWB: Social/family well-being; TOI: Trial outcome index; VAS: Visual analog scale

\section{Acknowledgements}

This study was sponsored by Gilead Sciences, Inc. (Foster City, CA; and Seattle, WA). The authors would like to thank Henry Adewoye and Yeonhee Kim, former employees of Gilead Sciences, Inc., for their invaluable input on the early drafts of this manuscript. Medical writing and editorial support were provided by Ewa Wandzioch, PhD; and Meryl Gersh, PhD, of AlphaBioCom, LLC, King of Prussia, PA, and was funded by Gilead Sciences, Inc.

\section{Authors' contributions}

All authors made substantial contributions to the acquisition, analysis, and/or interpretation of the data, provided critical feedback on manuscript content, provided final approval for submission of the manuscript to this journal, and have agreed to be accountable for all aspects of the work.

\section{Funding}

This study was funded by Gilead Sciences, Inc.

\section{Availability of data and materials}

The data that support the findings of this study are available from Gilead Sciences, Inc., but restrictions apply to the availability of these data, which were used under license for the current study, and so are not publicly available. Data are however available from the authors upon reasonable request and with permission of Gilead Sciences, Inc.

\section{Ethics approval and consent to participate}

The study protocols were approved by the Institutional Review Boards at each study site. The trial was conducted according to the principles of Good Clinical Practice and the Declaration of Helsinki. All patients provided written informed consent.

Consent for publication

Not applicable. 


\section{Competing interests}

MM reports research grants, personal fees, and nonfinancial support from Gilead Sciences, Inc;; as well as personal fees from Janssen, Roche, and Novartis. Al, ASP, ME, and WJ report institutional research grants from Gilead Sciences, Inc. TR reports research support and personal fees from Gilead Sciences, Inc. MW reports nothing to disclose. JD reports consulting and lecturing fees from Gilead Sciences, Inc.; Janssen; Roche; and GSK-Novartis. FM reports personal fees from Celgene; Genentech/Roche; Gilead Sciences, Inc.; and Janssen. AS reports honoraria from AbbVie; Gilead Sciences, Inc.; Janssen; and Roche; and nonrestricted educational grants from Gilead Sciences, Inc.; and Janssen. HE reports honoraria from Gilead Sciences, Inc.; AbbVie; Genentech; Pharmacyclics; and Janssen; consulting or advisory roles with Gilead Sciences, Inc.; AbbVie; Genentech; Pharmacyclics; and Janssen; speakers' bureau fees from Gilead Sciences, Inc.; AbbVie; Genentech; Pharmacyclics; and Takeda; and research funding from Gilead Sciences, Inc.; AbbVie; Genentech; Pharmacyclics; Roche; Celgene; Novartis; Kite; GlaxoSmithKline; AstraZeneca; ATARA; BeiGene; TG Therapeutics; and Verastem. SS is an employee of Gilead Sciences, Inc.; and may hold stock. JCB reports consulting or advisory roles with Pharmacyclics/AbbVie; Janssen; Genentech; and Gilead Sciences, Inc.; honoraria from Janssen; and institutional research funding from Pharmacyclics/AbbVie and Gilead Sciences, Inc. ADZ reports honoraria from Genentech/Roche; Gilead Sciences, Inc.; Celgene; Janssen; Amgen; Novartis; and Adaptive Biotech; consulting or advisory roles with Genentech/Roche; Gilead Sciences, Inc.; Celgene; Janssen; Amgen; Novartis; Adaptive Biotechnologies; and MEI Pharma; research funding from MEI Pharma; Genentech/Roche; Gilead Sciences, Inc.; BeiGene; and AbbVie; travel, accommodations, and expenses paid by Roche; Gilead Sciences, Inc.; and Celgene; and acting as a Data Monitoring Committee Chair for BeiGene.

\section{Author details}

'Department of Hematology, Niguarda Cancer Center, Niguarda Hospital, Piazza Ospedale Maggiore, 3, 20162 Milan, Italy. ${ }^{2}$ Department of Hematology, Faculty of Medicine, University of Debrecen, Debrecen, Hungary. ${ }^{3}$ Department of Hematology, Medical University of Lodz and Copernicus Memorial Hospital, Lodz, Poland. ${ }^{4}$ Department of Hematology, Ryazan Regional Clinical Hospital, Ryazan, Russia. ${ }^{5}$ Department Hemato-Oncology and Bone Marrow Transplantation, Medical University of Lublin, Lublin, Poland. ${ }^{6}$ Department of Hematology, Somogy County Kaposi Mor Hospital, Kaposvar, Hungary. ${ }^{7}$ Department of Hematology, Hospital Clínic, Barcelona, Spain. ${ }^{8}$ Maria Sklodowska Curie Institute of Oncology, Krakow, Poland. ${ }^{9} \mathrm{CHRU}$ Lille, Unité GRITA, Department of Hematology, Université de Lille, Lille, France. ${ }^{10}$ University of Oxford, Oxford Cancer and Haematology Centre, Churchill Hospital, Oxford, UK. "11Division of Hematology-Oncology, David Geffen School of Medicine at UCLA, Los Angeles, CA, USA. ${ }^{12}$ Gilead Sciences, Inc., Foster City, CA, USA. ${ }^{13}$ Zucker School of Medicine at Hofstra/Northwell, Hempstead, NY, USA. ${ }^{14}$ Memorial Sloan Kettering Cancer Center, New York, NY, USA.

Received: 28 June 2019 Accepted: 11 October 2019 Published online: 15 November 2019

\section{References}

1. SEER Cancer statistics review, 1975-2015. Available at https://seer.cancer. gov/statfacts/html/clyl.html. Accessed 22 Oct 2018.

2. Chronic Lymphocytic Leukemia/Small Lymphocytic Lymphoma. NCCN Clinical Practice Guidelines in Oncology 2017;Version 1.2017-September 28, 2016.

3. Badoux XC, Keating MJ, Wierda WG. What is the best frontline therapy for patients with CLL and 17p deletion? Curr Hematol Malig Rep. 2011;6:36-46.

4. Dicker F, Herholz H, Schnittger S, Nakao A, Patten N, Wu L, et al. The detection of TP53 mutations in chronic lymphocytic leukemia independently predicts rapid disease progression and is highly correlated with a complex aberrant karyotype. Leukemia. 2009;23:117-24.

5. Dohner $H$, Stilgenbauer $S$, Benner A, Leupolt E, Krober A, Bullinger $L$, et al. Genomic aberrations and survival in chronic lymphocytic leukemia. N Engl J Med. 2000;343:1910-6.

6. Stilgenbauer S, Sander S, Bullinger L, Benner A, Leupolt E, Winkler D, et al. Clonal evolution in chronic lymphocytic leukemia: acquisition of high-risk genomic aberrations associated with unmutated $\mathrm{VH}$, resistance to therapy, and short survival. Haematologica. 2007;92:1242-5.
7. Shanafelt T. Treatment of older patients with chronic lymphocytic leukemia: key questions and current answers. Hematol Am Soc Hematol Educ Program. 2013;2013:158-67.

8. Smolej L. How I treat elderly or comorbid patients with chronic lymphocytic leukemia. Acta Med (Hradec Kralove). 2010;53:213-20.

9. Smolej L. Therapy of elderly/comorbid patients with chronic lymphocytic leukemia. Curr Pharm Des. 2012;18:3399-405.

10. National Cancer Institute: Surveillance Epidemiology and End Results (SEER) program. SEER Stat Fact Sheets: Chronic Lymphocytic Leukemia; 2011. Available at http://seer.cancer.gov/statfacts/html/clyl.html. Accessed 12 Feb 2016.

11. Veliz M, Pinilla-lbarz J. Treatment of relapsed or refractory chronic lymphocytic leukemia. Cancer Control. 2012;19:37-53.

12. Shanafelt TD, Bowen D, Venkat C, Slager SL, Zent CS, Kay NE, et al. Quality of life in chronic lymphocytic leukemia: an international survey of 1482 patients. Br J Haematol. 2007;139:255-64.

13. Holtzer-Goor KM, Schaafsma MR, Joosten P, Posthuma EF, Wittebol S, Huijgens PC, et al. Quality of life of patients with chronic lymphocytic leukaemia in the Netherlands: results of a longitudinal multicentre study. Qual Life Res. 2015;24:2895-906.

14. Molica S. Quality of life in chronic lymphocytic leukemia: a neglected issue. Leuk Lymphoma. 2005;46:1709-14.

15. Basch E, Abernethy AP, Mullins CD, Reeve BB, Smith ML, Coons SJ, et al. Recommendations for incorporating patient-reported outcomes into clinical comparative effectiveness research in adult oncology. J Clin Oncol. 2012;30: 4249-55.

16. Garcia SF, Cella D, Clauser SB, Flynn KE, Lad T, Lai JS, et al. Standardizing patient-reported outcomes assessment in cancer clinical trials: a patientreported outcomes measurement information system initiative. J Clin Oncol. 2007;25:5106-12.

17. Hallek M, Cheson BD, Catovsky D, Caligaris-Cappio F, Dighiero G, Dohner H, et al. Guidelines for the diagnosis and treatment of chronic lymphocytic leukemia: a report from the International Workshop on Chronic Lymphocytic Leukemia updating the National Cancer Institute-Working Group 1996 guidelines. Blood. 2008;111:5446-56.

18. Cannella L, Caocci G, Jacobs M, Vignetti M, Mandelli F, Efficace F. Healthrelated quality of life and symptom assessment in randomized controlled trials of patients with leukemia and myelodysplastic syndromes: what have we learned? Crit Rev Oncol Hematol. 2015;96:542-54.

19. Catovsky D, Richards S, Matutes E, Oscier D, Dyer MJ, Bezares RF, et al. Assessment of fludarabine plus cyclophosphamide for patients with chronic lymphocytic leukaemia (the LRF CLL4 trial): a randomised controlled trial. Lancet. 2007;370:230-9.

20. Eichhorst BF, Busch R, Obwandner T, Kuhn-Hallek I, Herschbach $P$, Hallek M, German CLL Study Group. Health-related quality of life in younger patients with chronic lymphocytic leukemia treated with fludarabine plus cyclophosphamide or fludarabine alone for first-line therapy: a study by the German CLL Study Group. J Clin Oncol. 2007; 25:1722-31.

21. Robak T, Warzocha K, Govind Babu K, Kulyaba Y, Kuliczkowski K, Abdulkadyrov K, et al. Health-related quality of life and patient-reported outcomes of ofatumumab plus fludarabine and cyclophosphamide versus fludarabine and cyclophosphamide in the COMPLEMENT 2 trial of patients with relapsed CLL. Leuk Lymphoma. 2017;58:1598-606.

22. ZYDELIG (idelalisib) Prescribing Information. Gilead Sciences, Inc., Foster City, CA, USA. Available at http://www.gilead.com/ /media/Files/pdfs/ medicines/oncology/zydelig/zydelig_pi.pdf 2018;2016.

23. Zelenetz AD, Barrientos JC, Brown JR, Coiffier B, Delgado J, Egyed M, et al. Idelalisib or placebo in combination with bendamustine and rituximab in patients with relapsed or refractory chronic lymphocytic leukaemia: interim results from a phase 3, randomised, double-blind, placebo-controlled trial. Lancet Oncol. 2017;18:297-311.

24. Brucker PS, Yost K, Cashy J, Webster K, Cella D. General population and cancer patient norms for the Functional Assessment of Cancer TherapyGeneral (FACT-G). Eval Health Prof. 2005;28:192-211.

25. Cella DF, Tulsky DS, Gray G, Sarafian B, Linn E, Bonomi A, et al. The Functional Assessment of Cancer Therapy scale: development and validation of the general measure. J Clin Oncol. 1993;11:570-9.

26. Victorson D, Barocas J, Song J, Cella D. Reliability across studies from the Functional Assessment of Cancer Therapy-General (FACT-G) and its subscales: a reliability generalization. Qual Life Res. 2008;17:1137-46. 
27. Webster K, Chivington K, Shonk C, Eremenco S, Yount S, Hahn E. Measuring quality of life (QOL) among patients with leukemia: the Functional Assessment of Cancer Therapy- Leukemia (FACT-LEU). Qual Life Res. 2002;11: Abstract 678 .

28. Cella D, Jensen SE, Webster K, Hongyan D, Lai JS, Rosen S, et al. Measuring health-related quality of life in leukemia: the Functional Assessment of Cancer Therapy - Leukemia (FACT-Leu) questionnaire. Value Health. 2012;15:1051-8.

29. EuroQol G. EuroQol-a new facility for the measurement of health-related quality of life. Health Policy. 1990;16:199-208.

30. Rabin R, de Charro F. EQ-5D: a measure of health status from the EuroQol group. Ann Med. 2001;33:337-43.

31. FACIT.org, 2015. http://www.facit.org/FACITOrg/Questionnaires. Accessed 09 Mar 2017

32. EuroQol Research Foundation. EQ-5D-5L User Guide. Basic information on how to use the EQ-5D-5L instrument version 2.1. Available at https://euroqol.org/wp-content/uploads/2016/09/EQ-5D-5L_UserGuide_2 015.pdf. 2015

33. Shaw JW, Johnson JA, Coons SJ. US valuation of the EQ-5D health states: development and testing of the D1 valuation model. Med Care. 2005:43:203-20

34. Pickard AS, Neary MP, Cella D. Estimation of minimally important differences in EQ-5D utility and VAS scores in cancer. Health Qual Life Outcomes. 2007:5:70

35. Furman RR, Sharman JP, Coutre SE, Cheson BD, Pagel JM, Hillmen P, et al. Idelalisib and rituximab in relapsed chronic lymphocytic leukemia. N Engl J Med. 2014;370:997-1007.

36. Jones JA, Robak T, Brown JR, Awan FT, Badoux X, Coutre S, et al. Efficacy and safety of idelalisib in combination with ofatumumab for previously treated chronic lymphocytic leukaemia: an open-label, randomised phase 3 trial. Lancet Haematol. 2017:4:e114-e26.

37. Cramer P, Fraser G, Santucci-Silva R, Grosicki S, Dilhuydy MS, Janssens A, et al. Improvement of fatigue, physical functioning, and well-being among patients with severe impairment at baseline receiving ibrutinib in combination with bendamustine and rituximab for relapsed chronic lymphocytic leukemia/small lymphocytic lymphoma in the HELIOS study. Leuk Lymphoma. 2018:59:2075-84.

38. Hillmen P, Janssens A, Babu KG, Kloczko J, Grosicki S, Manson S, et al. Health-related quality of life and patient-reported outcomes of ofatumumab plus chlorambucil versus chlorambucil monotherapy in the COMPLEMENT 1 trial of patients with previously untreated CLL. Acta Oncol. 2016;55:1115-20.

\section{Publisher's Note}

Springer Nature remains neutral with regard to jurisdictional claims in published maps and institutional affiliations.

Ready to submit your research? Choose BMC and benefit from:

- fast, convenient online submission

- thorough peer review by experienced researchers in your field

- rapid publication on acceptance

- support for research data, including large and complex data types

- gold Open Access which fosters wider collaboration and increased citations

- maximum visibility for your research: over $100 \mathrm{M}$ website views per year

At $\mathrm{BMC}$, research is always in progress.

Learn more biomedcentral.com/submissions 